\title{
Use of antidepressant
}

\section{serotoninergic medications}

and cardiac valvulopathy: a nested case-control study in the health improvement

\section{network (THIN) database}

Francesco Lapi, ${ }^{1,2}$ Federica Nicotra, ${ }^{3,4}$ Lorenza Scotti, ${ }^{3}$

Alfredo Vannacci, ${ }^{1}$ Mary Thompson, ${ }^{5}$ Francesco Pieri, ${ }^{6}$

Niccolò Mugelli, ${ }^{1}$ Antonella Zambon, ${ }^{3}$ Giovanni Corrao, ${ }^{3}$

Alessandro Mugelli ${ }^{1} \&$ Annalisa Rubino ${ }^{1}$

${ }^{1}$ Department of Preclinical and Clinical Pharmacology 'Mario Aiazzi Mancini', University of Florence,

Florence, Italy, ${ }^{2}$ Department of Epidemiology, Biostatistics, and Occupational Health, McGill University,

Montreal, Quebec, Canada, ${ }^{3}$ Unit of Biostatistics and Epidemiology, Department of Statistics, University

of Milan-Bicocca, Milan, Italy, ${ }^{4}$ Department of Quantitative Methods for Economics and Business,

University of Milano-Bicocca, Milan, Italy, ${ }^{5}$ Cegedim Strategic Data Medical Research Ltd, London, UK

and ${ }^{6}$ Operative Unit of Cardiology III, Azienda Ospedaliero Universitaria Careggi, Florence, Italy

\section{WHAT IS ALREADY KNOWN ABOUT}

\section{THIS SUBJECT}

- Some medications can induce cardiac valvulopathy by acting on $5-\mathrm{HT}_{2 \mathrm{~B}}$ receptors. - Antidepressant serotoninergic medications may activate $5-\mathrm{HT}_{2 \mathrm{~B}}$ receptors via a direct or an indirect mechanism.

\section{WHAT THIS STUDY ADDS}

- These data do not appear to support an association between exposure to antidepressant serotoninergic medications and an increased risk of cardiac valvulopathy.

\section{AIMS}

To quantify the risk of cardiac valvulopathy (CV) associated with the use of antidepressant serotoninergic medications (SMs).

\section{METHODS}

We conducted a case-control study nested in a cohort of users of antidepressant SMs selected from The Health Improvement Network database. Patients who experienced a CV event during follow-up were cases. Cases were ascertained in a random sample of them. Up to 10 controls were matched to each case by sex, age, month and year of the study entry. Use of antidepressant SMs during follow-up was defined as current (the last prescription for antidepressant SMs occurred in the 2 months before the CV event), recent (in the 2-12 months before the $\mathrm{CV}$ event) and past (>12 months before the CV event). We fitted a conditional regression model to estimate the association between use of antidepressant SMs and the risk of CV by means of odds ratios (ORs) and corresponding $95 \%$ confidence intervals (Cls). Sensitivity analyses were conducted to test the robustness of our results.

\section{RESULTS}

The study cohort included 752945 subjects aged 18-89 years. Throughout follow-up, 1663 cases (incidence rate: 3.4 per 10000 person-years) of $\mathrm{CV}$ were detected and were matched to 16566 controls. The adjusted OR ( $95 \% \mathrm{Cl}$ ) for current and recent users compared with past users of antidepressant SMs were $1.16(0.96-1.40)$ and $1.06(0.93-1.22)$, respectively. Consistent effect estimates were obtained when considering cumulative exposure to antidepressant SMs during follow-up.

\section{CONCLUSIONS}

These results would suggest that exposure to antidepressant SMs is not associated with an increased risk of $\mathrm{CV}$. 


\section{Introduction}

Since 1960, some pharmacological treatments have been causally associated with cardiac valvulopathy (CV), including ergot derivatives used for the treatment of migraine, phentermine and phenfluramine [1-3] used to treat obesity and the antiparkinson drugs pergolide and carbergoline $[4,5]$.

Bench-to-bed evidence suggests that the pharmacological mechanisms of such iatrogenic disease involve the activation of $5-\mathrm{HT}_{2 B}$ receptors. In vitro data show that the stimulation of these serotonin receptors can upregulate the transforming growth factor $\beta$, which, in turn, induces hypertrophy of cardiomyocytes and proliferation of fibroblasts $[6,7]$. The serotonin agonism on the $5-\mathrm{HT}_{2 \mathrm{~B}}$ receptor has been proved to mediate the differentiation of adult stem cells into valvular interstitial cells [8]. Further studies indicate that long-term administration of serotonin can cause fibrotic heart lesions in animal models [9], while persistent pulmonary hypertension and heart malformations are likely to occur in newborns, following exposure to antidepressants in pregnancy [10].

In line with these data, there is clinical evidence on the role of $5-\mathrm{HT}_{2 \mathrm{~B}}$ receptors in valve fibrosis of carcinoid heart disease patients, in whom overproduction of serotonin by cancer metastases leads to the onset of CV [7]. Furthermore, a population-based study by Schade and colleagues [4] shows that among the antiparkinson medications those with a higher affinity for $5-\mathrm{HT}_{2 B}$ receptors (i.e. pergolide and carbergoline) are associated with an increased risk of $\mathrm{CV}$, while dopamine agonists with low affinity for $5-\mathrm{HT}_{2 \mathrm{~B}}$ receptors do not show such an association.

On the basis of these findings, it can be argued that medications acting at $5-\mathrm{HT}_{2 \mathrm{~B}}$ receptors should be investigated during the pre- and post-authorization phases of a product life cycle to assess any association with CV $[8,11]$. The objective of this study was therefore to quantify the risk of CV associated with the use of antidepressant serotoninergic medications (SMs) that activate $5-\mathrm{HT}_{2 \mathrm{~B}}$ receptors with a direct or an indirect mechanism. These medications include selective serotonin reuptake inhibitors (SSRIs), tricyclic antidepressants (TCAs), monoamine oxidase inhibitors (MAOI) and other antidepressant SMs (e.g. venlafaxine).

\section{Methods}

\section{Data source and study design}

The data used for this study were obtained from The Health Improvement Network (THIN) database. The network collects pseudo-anonymized patient data in a non-interventional way from the daily record keeping of a panel of primary care practices nationwide $(3$ million patients) in the UK. Patient-level medical records in THIN include demographic and comprehensive clinical informa- tion, such as diagnoses and symptoms, prescriptions, diagnostic tests, referrals and hospitalization, and other healthrelevant data, including the physician's free-text notes. The database also includes an area-based indicator of patients' socioeconomic circumstances. Each enumeration district (around 150 households) is assigned a Townsend deprivation score. These districts are divided into national fifths, and patients are assigned a quintile score according to the enumeration district in which they live. As general practitioners play a gatekeeper role within the National Health Service in the UK, electronic medical records kept in primary care represent a comprehensive medical history of patients, ideal for population-based characterization of therapeutic risk [12].

Within the THIN database, we conduced a matched case-control study nested in the cohort of new users of antidepressant SMs. The study was approved by the Cambridgeshire 4 Research Ethics Committee in the UK (Ref: 08/H0305/59).

\section{Cohort selection}

All patients aged 18-89 years who received at least one prescription of antidepressant SMs (i.e. SSRI, TCA, MAOI, other antidepressant SMs) during the period from 1 January 1990 to 30 June 2007 were selected. The date of their first recorded prescription of an antidepressant SM (cohort entry date) was considered the start of follow-up.

Patients were excluded from the cohort if any of the following criteria were applied: (i) the patient was registered as permanent at the practice for less than 6 months before the first antidepressant SM prescription, in order to ensure that all patients had a sufficient record from which to derive the covariates; (ii) the patient had less than 12 months longitudinal record after cohort entry, in order to ensure that all patients could be exposed for a period deemed to be necessary to induce valvulopathy [13]; (iii) the patient had a prescription for selected medications known to be associated with CV onset (fenfluramine, dexfenfluramine, phentermine, ergotamine, dihydroergotamine, methysergide, bromocriptine, cabergoline or pergolide); (iv) the patient had a heart murmur diagnosis associated with an echocardiography ( \pm 6 months from the date of heart murmur recording) or a CV diagnosis and/or specific surgical procedure at any time before the cohort entry; and (v) the patient had a diagnosis of cardiac prolapse, dilated cardiomiopathy, endocarditis and myocarditis, congenital or rheumatic cardiopathy, and carcinoid syndrome or with an indication of leisure drug use or drug abuse/addiction within the 12 month period before the cohort entry.

Each member of the cohort accumulated person-years of follow-up from the cohort entry until the earliest of the following events: a CV diagnosis; an exclusion event as for the third exclusion criteria occurring during follow-up (with the exception of congestive heart failure, which may precede a diagnosis of $\mathrm{CV}$ ); end of patient's record (i.e. 
death, end of registration with the practice or cessation of the contribution of data to THIN); or end of the study period (30 June 2007).

\section{Selection of cases and controls}

Potential cases of $\mathrm{CV}$ were defined on the basis of electronic coding for symptoms, diagnoses and referral, by means of the Read code Medical Dictionary. Codes for cardiac valve surgery and CV diagnosis were combined with codes for echocardiography within 6 months from the CV event. Coding for heart failure was not included in the definition of CV. Furthermore, codes defining valvular prolapses, rheumatic diseases and endocarditis with valvular involvement were not considered because of the known bacterial aetiology of these diseases, the established lack of relationship with drug-induced valve disorders, and because of their high prevalence in the general population [14].

For each case, up to 10 controls were randomly selected among the cohort patients. Controls were matched to their respective case by sex, age ( \pm 3 years), and month and year of the cohort entry, and were at risk for the outcome at the time of the matched case event (index date).

For each case, the date of CV diagnosis was the index date. The same date was assigned as the index date to the respective matched controls.

\section{Case ascertainment}

A random sample of 304 potential CV cases was extracted for the ascertainment process. The sample size was calculated assuming a positive predictive value (PPV) of $80 \%$, a maximum error accepted for PPV of 5\%, a power of $90 \%$ and a type I error of $5 \%$. Confirmation of a CV diagnosis was based on the combination of Read codes with general practitioners' verbatim, which was recorded in anonymized physician's free-text notes. The notes were manually reviewed by two cardiologists, blinded to each other and towards the study question. All clinical information available within 6 months from the date of a qualifying CV code was reviewed. Whenever there was disagreement, consensus was reached by means of an open discussion moderated by a third clinician.

\section{Exposure assessment}

Exposure to the drugs of interest was based on prescription records, assuming the actual use of the prescribed drugs. All prescription records of antidepressant SMs for cases and controls from the date of cohort entry to the index date were extracted. The duration of pharmacotherapy was computed, considering the strength (e.g. overall milligrams) of each prescription divided by the prescribed daily dose (PDD), as recorded in the database. Use of antidepressant SMs was defined as 'current' if the date of the last prescription was within 2 months from the index date, and as 'recent' or 'past' if the last antidepressant SM prescription was dated between 2 and 12 months or more than 12 months before index date, respectively.
Cumulative exposure to antidepressant SMs was constructed by summing up the days of medication use and was categorized as a cumulative duration of $<1,1-6,7-12$, 13-24 and $>24$ months.

Adherence to the treatment with antidepressant SMs during follow-up was expressed as proportion of days covered (PDC), which derived from dividing the cumulative days of use of antidepressant SMs by the length of followup. Progressively growing adherence to antidepressant SMs was categorized as a PDC value $<25,25-49,50-74$ and $\geq 75 \%$. For those prescriptions where no PDD record was available, the most frequent value being prescribed in the database for the same molecule was imputed.

\section{Covariates}

Demographic characteristics (age, sex) and starting class of antidepressant SMs were assessed from the patients' records at cohort entry. The starting class of antidepressant SMs were: SSRI, TCA, MAOI, other antidepressant SMs (e.g. venlafaxine) or any combination of those classes.

Other relevant co-morbidities, such as diabetes, coronary artery disease, left ventricular hypertrophy, atherosclerosis, stroke, renal insufficiency, hyperlipidaemia and hypertension, were accounted for any time before the index date. Hypertension was defined on the basis of medical coding, including codes that would indicate a value of systolic and diastolic blood pressure $>140$ and $>90 \mathrm{mmHg}$, respectively. Myocardial infarction events were considered only if they occurred in the year before the index date.

The smoking status and the body mass index (BMI) were evaluated based on the last available measurement before the index date. The BMI was categorized as follows: $<20,20-24,25-29,30-34,35-39$ and $\geq 40 \mathrm{~kg} \mathrm{~m}^{-2}$, while the smoking status was current, former and never smoker.

The analyses also accounted for the number of times during the follow-up that the class of antidepressant SMs changed from the class of antidepressant SMs at entry and for the Townsend score. When more than one score was available for a patient, the score closest in time to the index date was considered. Patients with a missing value for BMI, smoking or Towsend score were not included in the models.

\section{Data analysis}

We estimated that a sample size of 1600 cases and 16000 controls would allow us to show as statistically significant $(P<0.05)$ any odds ratio $(O R) \geq 1.35$ with a power of $80 \%$. This evaluation of the precision was based on the assumption that prevalence of exposure (i.e. use of antidepressants) in the source population was 5\% [15].

Statistical tests and techniques, including $\chi^{2}$, its version for the trend, and linear regression model, were used as appropriate to test differences in demographic, clinical and other characteristics of cases and controls. 
Conditional logistic regression for matched data was fitted to estimate the OR and corresponding 95\% confidence intervals (Cls) for the associations between cumulative exposure to antidepressant SMs and risk of CV and between PDC categories and risk of CV, calculated for all users (i.e. without distinction between current, recent and past users) and also stratified for categories of use of antidepressant SMs (i.e. current, recent and past users considered separately).

The OR estimates were adjusted for selected covariates (i.e. starting antidepressant SM treatment, number of classes of antidepressant SMs prescribed during follow-up, selected co-morbidities and other clinical characteristics).

Sensitivity analyses were carried out by excluding the following subpopulations: (i) patients with $<1$ month of exposure; (ii) patients with $<2$ years of follow-up; (iii) cases of CV defined only by Read codes for CV diagnosis, without coding for related echocardiography ( \pm 6 month period from the index date).
The SAS statistical package was used for the analyses (SAS, version 9.0; SAS Institute, Cary, NC, USA). For all hypotheses tested, two-tailed $P$ values $<0.05$ were considered to be significant.

\section{Results}

From the THIN database, 881527 first-time users of antidepressant SMs between 1 Januray 1990 and 30 June 2007 were selected. Of these, 752945 patients met the study eligibility criteria (Figure 1).

The cohort accumulated 4.8 million person-years during follow-up, with a mean (SD) follow-up per patient of 5.98 (4.25) years. Throughout follow-up, 1663 CV cases were identified, with an incidence rate of 3.41 per 10000 person-years at risk. The ascertainment of a random sample of 304 potential cases showed a PPV of $91.4 \%$.

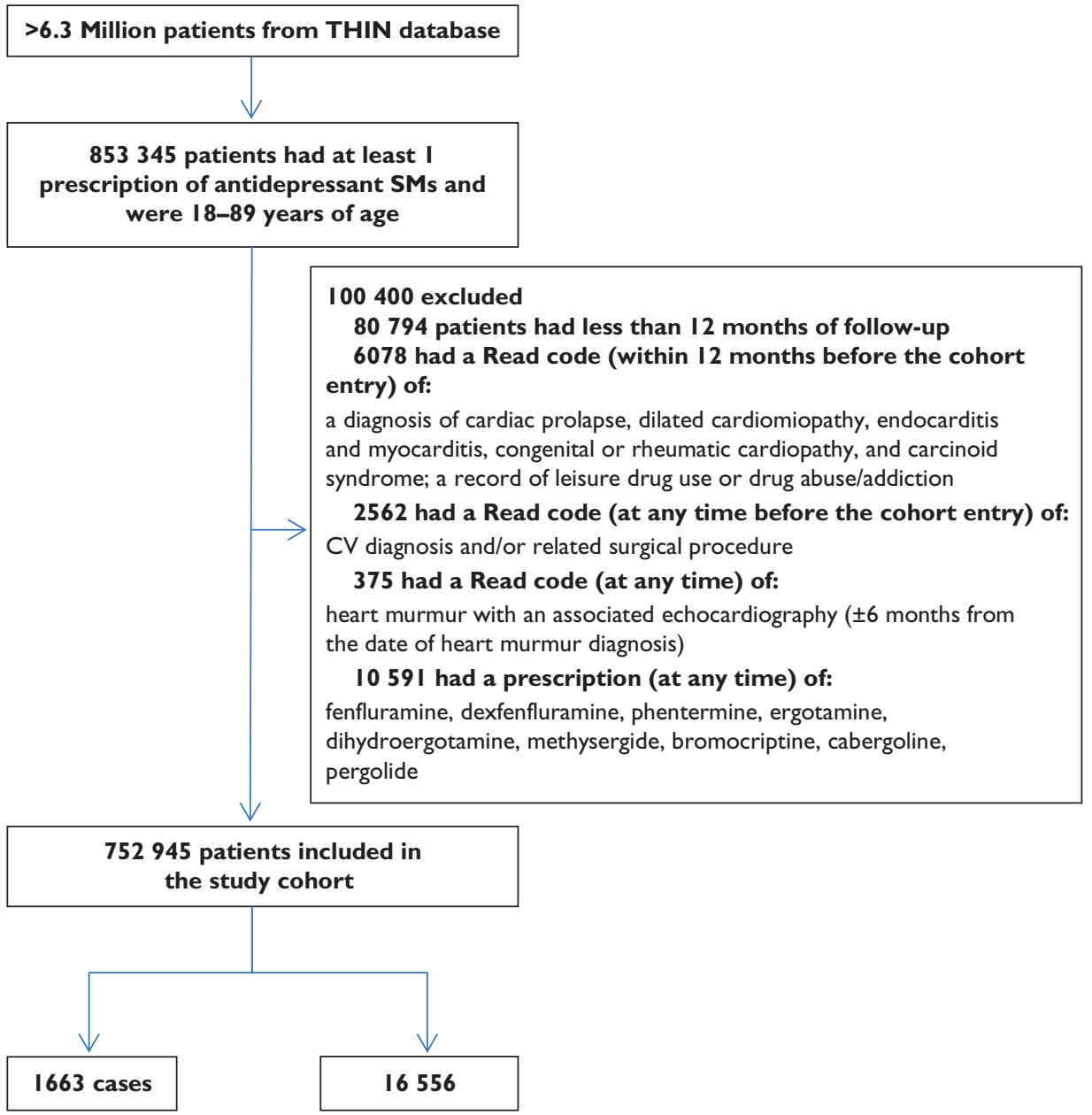

\section{Figure 1}

Process of cohort and case-control selection 


\section{Table 1}

Characteristics of cases and controls

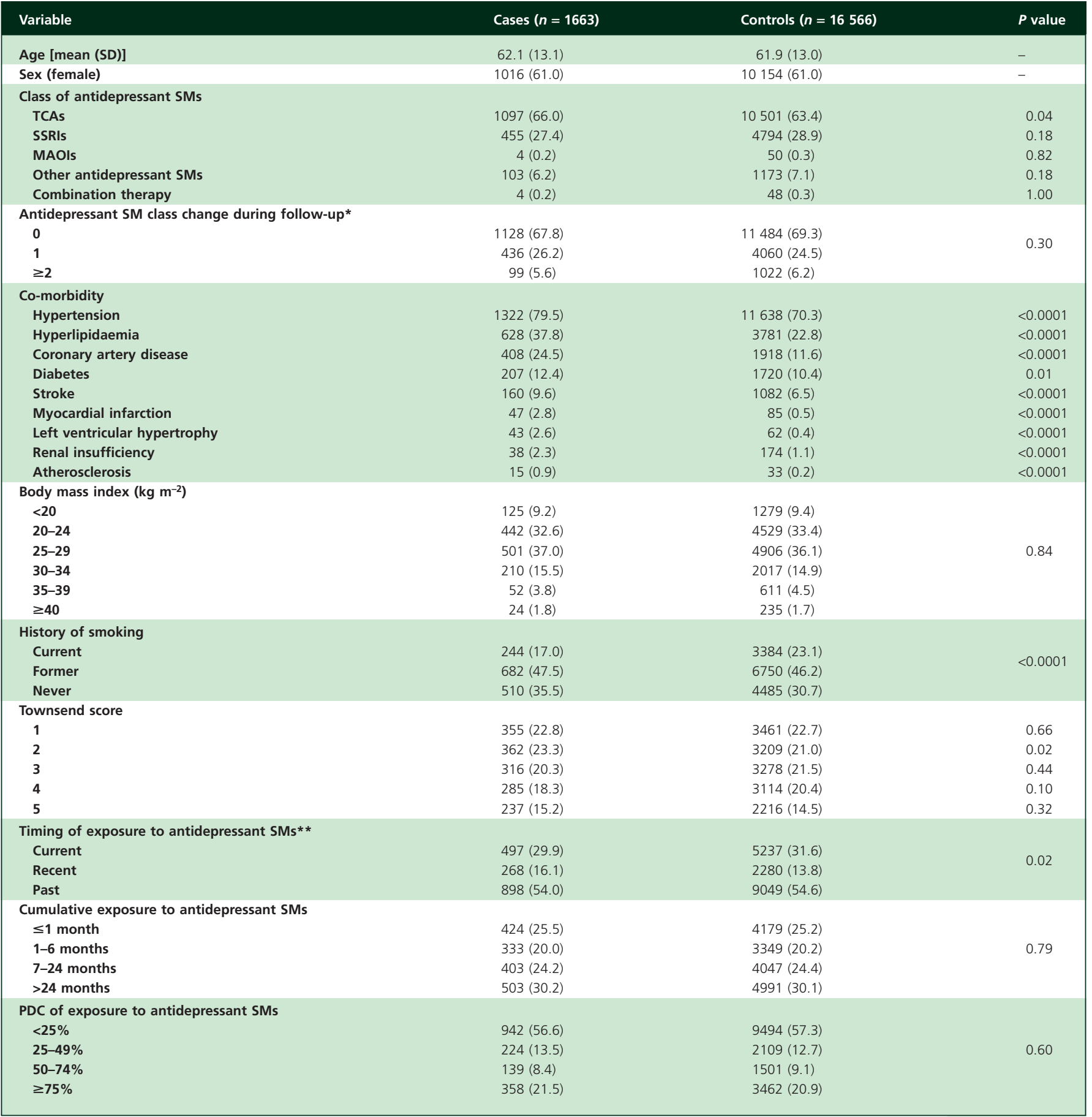

All values are reported as $n(\%)$. Abbreviations: MAOIs, monoamine oxidase inhibitors; PDC, proportion of days covered; SMs, serotoninergic medications; SSRIs, serotonin selective reuptake inhibitors; and TCAs, tricyclic antidepressants. *Number of antidepressant SM classes different from the cohort entry antidepressant SM class, changed during follow-up. ${ }^{*}$ Current use is defined as $<2$ months, including ongoing treatment at the index date; recent use, from 2 to 12 months before the index date; and past use, more than 12 months before the index date.

Each CV case was matched to up to 10 controls for a total of 16566 controls. Table 1 shows the distribution of demographic, clinical and lifestyle characteristics of cases and controls. The pattern of use of antidepressant SMs, including drug class at start of treatment and number of classes of antidepressant SMs used during follow-up, as well as recency, cumulative exposure and PDC of use of antidepressant SMs did not differ significantly between 


\section{Table 2}

Unadjusted and adjusted odds ratios (ORs) and corresponding 95\% confidence intervals (Cls) of cardiac valvulopathy risk associated with categories of cumulative use of antidepressant SMs among all, current, recent and past users

\begin{tabular}{|c|c|c|c|c|c|c|}
\hline & & \multicolumn{5}{|c|}{ Cumulative use of antidepressant SMs* } \\
\hline & & $<1$ month & 1-6 months & 7-12 months & 13-24 months & $>24$ months \\
\hline \multirow[t]{6}{*}{ All users } & Cases/controls & $422 / 4173$ & $335 / 3352$ & $201 / 1889$ & $201 / 2160$ & $504 / 4992$ \\
\hline & Unadjusted OR & 1.00 & 0.99 & 1.05 & 0.92 & 1.00 \\
\hline & $(95 \% \mathrm{Cl})$ & (reference) & $(0.85-1.15)$ & $(0.88-1.26)$ & $(0.77-1.10)$ & $(0.86-1.15)$ \\
\hline & Cases/controls & $315 / 3098$ & $242 / 2497$ & $149 / 1397$ & $147 / 1526$ & $385 / 3759$ \\
\hline & Adjusted OR & 1.00 & 0.95 & 0.99 & 0.91 & 0.99 \\
\hline & $(95 \% \mathrm{Cl})$ & (reference) & $(0.79-1.14)$ & $(0.79-1.23)$ & $(0.73-1.14)$ & $(0.83-1.19)$ \\
\hline \multirow[t]{6}{*}{ Current userst } & Cases/controls & $8 / 64$ & $21 / 268$ & $34 / 435$ & $87 / 975$ & $347 / 3495$ \\
\hline & Unadjusted OR & 1.00 & 0.77 & 0.77 & 0.88 & 0.98 \\
\hline & $(95 \% \mathrm{Cl})$ & (reference) & $(0.49-1.22)$ & $(0.54-1.11)$ & $(0.69-1.13)$ & $(0.84-1.15)$ \\
\hline & Cases/controls & $5 / 38$ & $17 / 191$ & $21 / 288$ & $64 / 653$ & $262 / 2626$ \\
\hline & Adjusted OR & 1.00 & 0.87 & 0.66 & 0.90 & 0.97 \\
\hline & $(95 \% \mathrm{Cl})$ & (reference) & $(0.51-1.49)$ & $(0.41-1.06)$ & $(0.66-1.22)$ & $(0.80-1.18)$ \\
\hline \multirow[t]{6}{*}{ Recent userst } & Cases/controls & $16 / 122$ & $60 / 528$ & $58 / 442$ & $43 / 423$ & $91 / 765$ \\
\hline & Unadjusted OR & 1.00 & 1.13 & 1.30 & 1.01 & 1.17 \\
\hline & $(95 \% \mathrm{Cl})$ & (reference) & $(0.84-1.50)$ & $(0.97-1.74)$ & $(0.73-1.40)$ & $(0.92-1.49)$ \\
\hline & Cases/controls & $7 / 86$ & $37 / 388$ & $45 / 329$ & 28/295 & $70 / 590$ \\
\hline & Adjusted OR & 1.00 & 0.88 & 1.22 & 0.82 & 1.14 \\
\hline & $(95 \% \mathrm{Cl})$ & (reference) & $(0.60-1.29)$ & $(0.85-1.76)$ & $(0.53-1.26)$ & $(0.85-1.53)$ \\
\hline \multirow[t]{6}{*}{ Past userst } & Cases/controls & 398/3987 & $254 / 2556$ & $109 / 1012$ & $71 / 762$ & $66 / 732$ \\
\hline & Unadjusted OR & 1.00 & 0.98 & 1.07 & 0.92 & 0.89 \\
\hline & $(95 \% \mathrm{Cl})$ & (reference) & $(0.83-1.16)$ & $(0.85-1.34)$ & $(0.71-1.21)$ & $(0.67-1.18)$ \\
\hline & Cases/controls & $303 / 2974$ & $188 / 1918$ & $83 / 780$ & $55 / 578$ & $53 / 543$ \\
\hline & Adjusted OR & 1.00 & 0.97 & 1.01 & 0.97 & 0.97 \\
\hline & $(95 \% \mathrm{Cl})$ & (reference) & $(0.79-1.19)$ & $(0.77-1.32)$ & $(0.71-1.34)$ & $(0.69-1.34)$ \\
\hline
\end{tabular}

*According to the time interval spent with available antidepressant SMs during follow-up categorized into four categories. $†$ According to the time interval since last antidepressant SM use before the index date. Current use is defined as $<2$ months, including ongoing treatment at the index date; recent use, from 2 to 12 months before the index date; and past use, more than 12 months before the index date. Estimates are adjusted for type of cohort entry antidepressant SM class (i.e. SSRIs, TCAs, MAOIs, other antidepressant SMs and combination of different antidepressant SM classes), number of antidepressant SM classes, different from the cohort entry antidepressant SM class, changed during follow-up, diabetes, hypertension, coronary artery disease, left ventricular hypertrophy, atherosclerosis, stroke, renal insufficiency, hyperlipidaemia, body mass index, smoking and Townsend score. The numbers of cases and controls are different between univariate and multivariate analysis because of missing values of the covariates.

cases and controls, while co-morbidities were more prevalent among cases. Cases and controls were significantly different with respect to their smoking status, while the distribution of their respective deprivation scores was comparable (Table 1).

Cumulative exposure to antidepressant SMs for 1-6, 7-12, 13-24 and >24 months compared with cumulative use of antidepressant SMs for $<1$ month did not appear to be associated with an increased risk of CV (Table 2). When cumulative duration of antidepressant SMs was stratified by recency of use (i.e. current, recent or past use), the OR estimates did not show any statistically significant association between use of antidepressant SMs and risk of CV (Table 2).

When PDC levels were considered, adjusted and unadjusted ORs for adherence at $25-49,50-74$ and $\geq 75 \%$ compared with $<25 \%$ did not show any statistically significant association between use of antidepressant SMs and onset of CV (Table 3). When results were stratified by recency of use, adherence $\geq 75 \%$ during recent use of antidepressant SMs was associated with a statistically significant increase of CV risk (OR 1.45; 95\% Cl 1.04-2.04; Table 3).

Sensitivity analyses showed consistent results with those reported in Tables 2 and 3 (data not shown).

\section{Discussion}

This study indicated that prolonged treatment with medications that directly or indirectly activate $5-\mathrm{HT}_{2 B}$ receptors was not associated with an increased risk of CV.

The population-based estimate of CV risk produced in this analysis is in line with data from the literature [4], which strengthens the validity of the case definition and the overall methodological approach used in this study. Furthermore, the operational change of case definition in the sensitivity analysis produced comparable results.

Although the evidence is inconsistent, it is generally accepted that high serum serotonin concentrations are needed, together with an exposure time from 6 to 11 months, in order to induce CV $[6,7,13]$. Within the cohort of users of antidepressant SMs, we identified a reference group in those patients exposed for $<1$ month of cumulative use of antidepressant SMs or only currently exposed to antidepressant SMs. Neither cumulative exposure up to $>24$ months nor past exposure with >12 months since last prescription of antidepressant SMs was significantly associated with an increased risk of CV. Lack of association appeared to be confirmed when exposure to antidepressant SMs was expressed as PDC. Thus, reproducibility of all 


\section{Table 3}

Unadjusted and adjusted odds ratios (ORs), and corresponding 95\% confidence intervals (Cls) of cardiac valvulopathy risk associated with categories of proportion of days covered (PDC) by antidepressant SMs among all, current, recent and past users

\begin{tabular}{|c|c|c|c|c|c|}
\hline & & \multicolumn{4}{|c|}{ PDC* } \\
\hline & & $<25 \%$ & $25-49 \%$ & $50-74 \%$ & $\geq 75 \%$ \\
\hline \multirow[t]{6}{*}{ All users } & Cases/controls & $942 / 9494$ & $224 / 2109$ & 139/1501 & $358 / 3462$ \\
\hline & Unadjusted OR & 1.00 & 1.07 & 0.93 & 1.04 \\
\hline & $(95 \% \mathrm{Cl})$ & (reference) & $(0.92-1.25)$ & $(0.77-1.12)$ & $(0.92-1.19)$ \\
\hline & Cases/controls & 709/7191 & 161/1539 & 105/1112 & $263 / 2435$ \\
\hline & Adjusted OR & 1.00 & 1.01 & 0.93 & 1.09 \\
\hline & $(95 \% \mathrm{Cl})$ & (reference) & $(0.84-1.22)$ & $(0.74-1.17)$ & $(0.93-1.28)$ \\
\hline \multirow[t]{5}{*}{ Current userst } & Cases/controls & $54 / 673$ & $72 / 764$ & $81 / 877$ & $290 / 2923$ \\
\hline & Adjusted OR & 1.00 & 0.95 & 0.93 & 1.00 \\
\hline & $(95 \% \mathrm{Cl})$ & (reference) & $(0.74-1.22)$ & $(0.74-1.18)$ & $(0.87-1.15)$ \\
\hline & Cases/controls & $44 / 504$ & $54 / 578$ & $58 / 651$ & $213 / 2063$ \\
\hline & $\begin{array}{l}\text { Adjusted OR } \\
(95 \% \mathrm{Cl})\end{array}$ & $\begin{array}{l}1.00 \\
\text { (reference) }\end{array}$ & $\begin{array}{l}0.89 \\
(0.65-1.20)\end{array}$ & $\begin{array}{l}0.89 \\
(0.66-1.20)\end{array}$ & $\begin{array}{l}1.03 \\
(087-1.23)\end{array}$ \\
\hline \multirow[t]{6}{*}{ Recent userst } & Cases/controls & $101 / 910$ & $65 / 538$ & $40 / 382$ & $62 / 450$ \\
\hline & Unadjusted OR & 1.00 & 1.23 & 1.06 & 1.38 \\
\hline & $(95 \% \mathrm{Cl})$ & (reference) & $(0.94-1.61)$ & $(0.76-1.47)$ & $(1.05-1.82)$ \\
\hline & Cases/controls & $69 / 701$ & $40 / 385$ & $32 / 279$ & $46 / 323$ \\
\hline & Adjusted OR & 1.00 & 0.93 & 1.06 & 1.45 \\
\hline & $(95 \% \mathrm{Cl})$ & (reference) & $(0.65-1.33)$ & $(0.71-1.57)$ & $(1.04-2.04)$ \\
\hline \multirow[t]{6}{*}{ Past userst } & Cases/controls & 782/7911 & $87 / 807$ & $18 / 242$ & $6 / 89$ \\
\hline & Unadjusted OR & 1.00 & 1.09 & 0.75 & 0.68 \\
\hline & $(95 \% \mathrm{Cl})$ & (reference) & $(0.86-1.37)$ & $(0.46-1.21)$ & $(0.30-1.55)$ \\
\hline & Cases/controls & $596 / 5986$ & $67 / 576$ & $15 / 182$ & $4 / 49$ \\
\hline & Adjusted OR & 1.00 & 1.19 & 0.87 & 0.86 \\
\hline & $(95 \% \mathrm{Cl})$ & (reference) & $(0.91-1.57)$ & $(0.50-1.52)$ & $(0.30-2.48)$ \\
\hline
\end{tabular}

*According to the time interval spent with available antidepressant SMs during follow-up categorized into four categories. †According to the time interval since last use of antidepressant SMs before the index date. Current use is defined as $<2$ months, including ongoing treatment at the index date; recent use, from 2 to 12 months before the index date; and past use, more than 12 months before the index date. Estimates are adjusted for type of antidepressant SM classes at cohort entry (i.e. SSRIs, TCAs, MAOIs, other antidepressant SMs and combination of different antidepressant SM classes), number of antidepressant SM classes (different from the antidepressant SM class at cohort entry) changed during follow-up, diabetes, hypertension, coronary artery disease, left ventricular hypertrophy, atherosclerosis, stroke, renal insufficiency, hyperlipidaemia, body mass index, smoking and Townsend score. The numbers of cases and controls are different between univariate and multivariate analysis because of missing values of the covariates.

analyses when exposure was modelled according to different recency or other parameters strengthened the study findings.

Only with the highest adherence to medications and when the use of antidepressant SMs extended to include the 2-12 months before the CV diagnosis was the risk of experiencing the event increased by $45 \%$ and statistical significance reached. These data would be in line with evidence in the carcinoid syndrome, where the time, as well as the intensity exerted by nonphysiological serotonin levels, is crucial in causing CV [16]. Nevertheless, the absence of an association among current users and the wide $\mathrm{Cls}$ seen in the recent use cannot exclude the possibility that the higher risk reported in this subgroup was due simply to random fluctuation.

Previous data have shown a lack of association between use of SSRIs and CV [17]. From the pharmacological perspective, several reasons may explain those results.

Although stimulation of the $5-\mathrm{HT}_{2 B}$ receptor subtype seems to be the main factor in the aetiology of druginduced valvulopathy $[4,8,11,18]$, serotonin does not appear to be the only mediator to be involved in the aetiology of $\mathrm{CV}$, and a complex interplay, comprising serotonin and other mediators (e.g. histamine, bradykinin), could cause cardiac complications in the carcinoid syndrome.

All the antidepressant SMs studied act by increasing the levels of serotonin, which is the endogenous agonist of $5-\mathrm{HT}_{2 \mathrm{~B}}$ receptors. However, increased blood and/or synaptic cleft concentrations of serotonin resulting from the pharmacological effects of antidepressant SMs might not be sufficient to increase the risk of CV. Indeed, in patients with carcinoid disease, the concurrent use of antidepressant SMs does not seem to induce serotoninergic syndrome and/or exacerbation of the carcinoid disease [19]. On the contrary, the direct binding of certain medications to 5 -HT receptors is more likely to have a causal association with $\mathrm{CV}$, because drugs known to cause CV have higher affinity towards the $5-\mathrm{HT}_{2 B}$ subtype. For instance, among antiparkinson agents, only cabergoline and pergolide with a proven affinity for $5-\mathrm{HT}_{2 \mathrm{~B}}$ receptors induced $\mathrm{CV}$. Lisuride and pramiprexole, with no affinity for $5-\mathrm{HT}_{2 B}$ receptors, were not associated with valve fibrosis [4]. In our study, only fluoxetine and trazodone have some (low) affinity for $5-\mathrm{HT}_{2 B}$ receptors [20-22]. A poor stimulating effect of the study drugs on this specific receptor subtype could explain the lack of association between antidepressant SMs and 
CV that we observed in most of our analyses. Genetic predisposition could be postulated as a potential risk factor for this disease. Indeed, genetic polymorphisms of 5-HT membrane transporters have been associated with variable pharmacological responsiveness to SSRIs, and not all polymorphisms would significantly increase the serotonin blood concentrations until the induction of CV [23]. As this study included a relatively small number of CV cases, of whom only a very small subgroup could possess polymorphism of 5-HT transporters associated with induction of $\mathrm{CV}$, this study might have insufficient statistical power to detect a significant association between use of antidepressant SMs and CV in all the stratified analyses.

From a clinical perspective, the lack of association we observed in this study could sound reassuring, particularly when accounting for the growing use of antidepressants in western countries [24]. However, a statistically significant increase of the CV risk was associated with the highest level of exposure during recent use of antidepressant SMs, which warrants further investigations. An effective pharmacotherapy of depression is based on different phases (acute, maintenance and continuation), and exposure to medications can reach a total duration of 48 months, in order to significantly reduce the risk of recurrences [25]. In this context, the results of this study bear clinical relevance, although the relationship between individual antidepressant SMs and cardiovascular disorders remains controversial $[26,27]$.

The major strength of our investigation is the large source population and study size, the longitudinal nature of patients' records, which allowed an evaluation of the remote use of the study drugs and access to comprehensive patients' medical histories, including smoking, BMI and Townsend score data, which allowed adjustment for numerous risk factors and potential confounders.

Nonetheless, the study size remained a limiting factor that prevented assessment of the association of CV with individual medicinal products or their combination. Several additional limitations should be also considered. First, valvulopathy has a subtle onset. For this reason, some events, being subclinical, could not have been captured by physicians' observations, while more severe cases are more likely to have been detected. Although the incidence of CV we computed was in line with data from the literature [4], it should be noted that there are large differences in the incidence of CV between primary care and the specialist setting $[28,29]$. Case definition was based on coded information, and no formal validation of individual cases was performed even if the expert review of a random sample of cases resulted in a high PPV and a sensitivity analysis supported the case definition.

In any retrospective analysis of medical records, assessment of exposure can be challenging. In this study, exposure to antidepressant SMs, including adherence, was assumed from prescription record extracts. Such an approach might have resulted in misclassification of the exposure and dilution of the association towards the null. In the light of these considerations, a possible association between use of antidepressant SMs and CV should not be excluded.

\section{Conclusions}

In this study, treatment with antidepressant SMs that directly or indirectly activate $5-\mathrm{HT}_{2 \mathrm{~B}}$ receptors did not appear to be associated with an increased risk of CV. From a clinical perspective, these results would sound encouraging, particularly when considering the increasing use of antidepressant SMs in western countries. Nevertheless, these results need to be confirmed, because a significant increase of the CV risk was observed with the highest degree of adherence in recent use of antidepressant SMs. The relationship between some antidepressant SMs and cardiovascular diseases remains controversial, and further investigation is warranted to clarify the association between use of individual medications that involve serotoninergic mechanisms and heart disorders.

\section{Competing Interests}

There are no competing interests to declare.

Francesco Lapi, Giovanni Corrao, Alessandro Mugelli, Alfredo Vannacci and Annalisa Rubino were responsible for initiating the study, including development of the study protocol development and analytical plan. Federica Nicotra, Lorenza Scotti and Antonella Zambon were responsible for data management and analysis. Mary Thompson provided expertise for optimization of the use of THIN data extracts. Francesco Pieri and Niccolò Mugelli reviewed clinical records of $C V$, including codes and free text for CV ascertainment. Francesco Lapi had full access to all data in the study and takes responsibility for the integrity of the data and the accuracy of the data analysis.

We are grateful to the general practitioners who contribute data to THIN. The data extracts from the THIN Database were kindly provided by Cegedim Strategic Data Medical Research Limited.

\section{REFERENCES}

1 From the Centers for Disease Control and Prevention. Cardiac valvulopathy associated with exposure to fenfluramine or dexfenfluramine: US Department of Health and Human Services interim public health recommendations, November 1997. JAMA 1997; 278: 1729-31.

2 Centers for Disease Control and Prevention (CDC). Cardiac valvulopathy associated with exposure to fenfluramine or dexfenfluramine: U.S. Department of Health and Human Services interim public health recommendations, November 1997. MMWR Morb Mortal Wkly Rep 1997; 46: 1061-6. 
3 Anchors M. Fluoxetine is a safer alternative to fenfluramine in the medical treatment of obesity. Arch Intern Med 1997; 157: 1270

4 Schade R, Andersohn F, Suissa S, Haverkamp W, Garbe E. Dopamine agonists and the risk of cardiac-valve regurgitation. N Engl J Med 2007; 356: 29-38.

5 Zanettini R, Antonini A, Gatto G, Gentile R, Tesei S, Pezzoli G. Valvular heart disease and the use of dopamine agonists for Parkinson's disease. N Engl J Med 2007; 356: 39-46.

6 Moller JE, Connolly HM, Rubin J, Seward JB, Modesto K, Pellikka PA. Factors associated with progression of carcinoid heart disease. N Engl J Med 2003; 348: 1005-15.

7 Druce M, Rockall A, Grossman AB. Fibrosis and carcinoid syndrome: from causation to future therapy. Nat Rev Endocrinol 2009; 5: 276-83.

8 Roth BL. Drugs and valvular heart disease. N Engl J Med 2007; 356: 6-9.

9 Gustafsson BI, Tommeras K, Nordrum I, Loennechen JP, Brunsvik A, Solligard E, Fossmark R, Bakke I, Syversen U, Waldum $\mathrm{H}$. Long-term serotonin administration induces heart valve disease in rats. Circulation 2005; 111: 1517-22.

10 Reis M, Kallen B. Delivery outcome after maternal use of antidepressant drugs in pregnancy: an update using Swedish data. Psychol Med 2010; 40: 1-11.

11 Andersohn F, Garbe E. Cardiac and noncardiac fibrotic reactions caused by ergot-and nonergot-derived dopamine agonists. Mov Disord 2009; 24: 129-33.

12 Lewis JD, Schinnar R, Bilker WB, Wang X, Strom BL. Validation studies of the health improvement network (THIN) database for pharmacoepidemiology research. Pharmacoepidemiol Drug Saf 2007; 16: 393-401.

13 Smith SA, Waggoner AD, de las Fuentes L, Davila-Roman VG. Role of serotoninergic pathways in drug-induced valvular heart disease and diagnostic features by echocardiography. J Am Soc Echocardiogr 2009; 22: 883-9.

14 Singh JP, Evans JC, Levy D, Larson MG, Freed LA, Fuller DL, Lehman B, Benjamin EJ. Prevalence and clinical determinants of mitral, tricuspid, and aortic regurgitation (the Framingham Heart Study). Am J Cardiol 1999; 83: 897-902.

15 Morgan O, Griffiths C, Majeed A. Antidepressant prescribing and changes in antidepressant poisoning mortality and suicide in England, 1993-2004. J Public Health (Oxf) 2008; 30: $60-8$.

16 Druce MR, Bharwani N, Akker SA, Drake WM, Rockall A, Grossman AB. Intra-abdominal fibrosis in a recent cohort of patients with neuroendocrine ('carcinoid') tumours of the small bowel. QJM 2010; 103: 177-85.

17 Mast ST, Gersing KR, Anstrom KJ, Krishnan KR, Califf RM, Jollis JG. Association between selective serotonin-reuptake inhibitor therapy and heart valve regurgitation. Am J Cardiol 2001; 87: 989-93.

18 Mekontso-Dessap A, Brouri F, Pascal O, Lechat P, Hanoun N, Lanfumey L, Seif I, Benhaiem-Sigaux N, Kirsch M, Hamon M, Adnot S, Eddahibi S. Deficiency of the 5-hydroxytryptamine transporter gene leads to cardiac fibrosis and valvulopathy in mice. Circulation 2006; 113: 81-9.

19 Williams MD, Dolenc TJ. Selective serotonin reuptake inhibitors and patients with carcinoid tumor. Psychosomatics 2005; 46: 370-2.

20 Rothman RB, Baumann MH. Serotonergic drugs and valvular heart disease. Expert Opin Drug Saf 2009; 8: 317-29.

21 Rothman RB, Baumann MH. Appetite suppressants, cardiac valve disease and combination pharmacotherapy. Am J Ther 2009; 16: 354-64.

22 Rothman RB, Baumann MH, Savage JE, Rauser L, McBride A, Hufeisen SJ, Roth BL. Evidence for possible involvement of $5-\mathrm{HT}(2 \mathrm{~B})$ receptors in the cardiac valvulopathy associated with fenfluramine and other serotonergic medications. Circulation 2000; 102: 2836-41.

23 Lotrich FE, Pollock BG, Ferrell RE. Polymorphism of the serotonin transporter: implications for the use of selective serotonin reuptake inhibitors. Am J Pharmacogenomics 2001; 1: 153-64.

24 Moore M, Yuen HM, Dunn N, Mullee MA, Maskell J, Kendrick T. Explaining the rise in antidepressant prescribing: a descriptive study using the general practice research database. BMJ 2009; 339: b3999.

25 Mann JJ. The medical management of depression. N Engl J Med 2005; 353: 1819-34.

26 Martinez C, Assimes TL, Mines D, Dell'aniello S, Suissa S. Use of venlafaxine compared with other antidepressants and the risk of sudden cardiac death or near death: a nested case-control study. BMJ 2010; 340: c249.

27 Monte S, Macchia A, Romero M, D'Ettorre A, Giuliani R, Tognoni G. Antidepressants and cardiovascular outcomes in patients without known cardiovascular risk. Eur J Clin Pharmacol 2009; 65: 1131-8.

28 Shiran A, Sagie A. Tricuspid regurgitation in mitral valve disease incidence, prognostic implications, mechanism, and management. J Am Coll Cardiol 2009; 53: 401-8.

29 lung B, Vahanian A. Epidemiology of valvular heart disease in the adult. Nat Rev Cardiol 2011; 8: 162-72. 